\title{
in-vitro phagocytosis and intracellular killing of pasteurella multocida b:2 by phagocytic cells of buffaloes
}

\begin{abstract}
Pasteurella multocida B:2 is a Gram-negative organism causing haemorrhagic septicaemia (HS) in buffaloes. It causes severe pulmonary infection, leading to infiltration of numerous macrophages and neutrophils. Despite the inflammatory response, buffaloes succumb to HS. This study aims to evaluate the in-vitro efficacy of macrophages and neutrophils of buffalo following exposure to P. multocida B:2. In-vitro infections were done using $10^{7} \mathrm{cfu} / \mathrm{ml}$ of $\mathrm{P}$. multocida B:2 for Group 1, Escherichia coli for Group 2 and Mannhaemia haemolytica A:2 for Group 3 cells. The inoculated cell cultures were harvested at 0, 30, 60 and 120 min postexposure and the phagocytic, killing and cell death rates were determined. Both phagocytosis and killing rates of all bacteria increased over time. Phagocytosis involved between $71 \%$ and $73 \%$ neutrophils and between $60 \%$ and $64 \%$ macrophages at $120 \mathrm{~min}$. Killing rate of all bacteria involved between $76 \%$ and $79 \%$ for neutrophils and between $70 \%$ and $74 \%$ for macrophages at $120 \mathrm{~min}$. Death rate of neutrophils ranged between 67\% in Group 3, and 88\% in Group 1 at 120 min, significantly ( $<$ 0.05) higher than Group 3 but insignificant $(p>0.05)$ than Group 2. Similar pattern was observed for death rate of macrophages. The phagocytosis and killing rates of P. multocida B:2 were similar to other bacterial species used in this study but more neutrophils and macrophages were dead following infection by P. multocida B:2 than M. haemolytica A:2.
\end{abstract}

Keyword: Buffalo; Cell death; Haemorrhagic septicaemia; Killing rate; Pasteurella multocida $\mathrm{B}: 2$; Phagocytosis 